\title{
FLUENT-based Wear Research for the Centrifugal Sewage Pump
}

\author{
Liqiang Zhang ${ }^{1,2, a}$, Shuaipeng Yang ${ }^{1,2, b}$, Sudan Huang ${ }^{1,2, c}$ \\ ${ }^{1}$ School of Energy and Power Engineering, Lanzhou University of Technology, Lanzhou 730050, \\ China \\ ${ }^{2}$ Wenzhou Academy of Pump and Valve Engineering, Lanzhou University of Technology, Wenzhou \\ 325105, China \\ azhangold@qq.com, b $18893493117 @ q q . c o m,{ }^{c} 18189699851 @ q q . c o m$
}

Keywords: sewerage pump, flow characteristics, granulometric distribution, numerical simulation.

\begin{abstract}
Adopting SIMPLE algorithm, k- $\varepsilon$-Ap turbulence model and tetrahedron non-structure grid simulates the flow field of sewerage pump in the Descartes reference frame. Through numerical simulation, flow characteristics and distribution rule of sold particles in sewerage pump are obtained, which supply can contribute to optimization design of centrifugal sewage pump.
\end{abstract}

\section{Introduction}

The centrifugal sewage pump is a kind of centrifugal pump which has little blades. It is suitable for the transmission of fluid medium contains solid particles below $80^{\circ} \mathrm{C}$. With good Non-clogging performance and simple manufacture, it has been widely used. Based on the internal flow of sewage pump is a complex three-dimensional flow, the flow discipline is not completely clear and the design theory is not perfect, so it is necessary to research on the internal flow of sewage pump.

In the solid-liquid two-phase flow, the common methods are Euler and Lagrange. In Euler method, solid particles are regarded as pseudo-fluid, and particles and fluid are considered as continuous medium with co-existence and mutual penetration. The flow of pseudo-fluid is described with conservation equation in the macroscopic continuum principle, while the flow interaction of solid-liquid two-phase is mainly indicated by solid viscosity and solid pressure. In Lagrange method, the liquid and solid particles are regarded as continuous medium and discrete system respectively. Investigate the motion of liquid in Euler coordinate, and research the motion of solid particles in Lagrange coordinate which means the particle trajectory model. Its interaction is mainly processed through the perfectly elastic collision model or particles discrete element method.

In this paper, the solid-liquid two-phase turbulent flow of centrifugal impeller for sewerage pump is simulated through FLUENT software, which adopts N-S equation, $\mathrm{k}-\varepsilon$-Ap turbulence model and SIMPLE algorithm. Through numerical simulation, flow characteristics and distribution rule of sold particles in sewerage pump are obtained, which can supply reference to optimization design of centrifugal sewage pump.

\section{The basic theory of turbulence model calculation model}

In this paper, the two-phase flow of centrifugal pump when transport the water and solid particles is simulated based on the following assumptions:

1. The flow is steady, three-dimensional, and isothermal with incompressible.

2. The shape of the solid particles remains unchanged during the motion.

3. There are no mass and energy exchange between the solid-liquid two-phase and two-phase.

Based on the above assumptions, The Euler model is used to analysis the centrifugal sewage pump with sparse two-phase medium.

Equations: the basic equations are as follows.

The continuity equation in Euler model is given by Eq.

$\nabla \cdot\left(\alpha_{p} \rho_{p} \vec{v}_{p}\right)=0$ 
The momentum equation in Euler model is given by Eq

$$
\begin{aligned}
& \nabla \cdot\left(\alpha_{p} \rho_{p} \vec{v}_{p} \vec{v}_{p}\right)=-\alpha_{p} \nabla p+\nabla \bar{\tau}+ \\
& \vec{R}_{L, S}+\alpha_{p} \rho_{p}\left(\vec{F}_{p}+\vec{F}_{l i f t, p}+\vec{F}_{v m, p}\right)
\end{aligned}
$$

Where, the index $\mathrm{P}$ of $\mathrm{L}$ and $\mathrm{S}$ denotes liquid and solid phase respectively. $\vec{R}_{L, S}$ denotes the inter phase force, and $\vec{R}_{L, S}=\alpha_{S} \rho_{S} f\left(\vec{v}_{L}-\vec{v}_{S}\right) / \tau_{S}, \tau_{S}$ (3) denotes the relaxation time of the particles, $\alpha_{p}$ denotes the volume fraction and $f=C_{D} R_{e} / 24$ (4), $C_{D}$ denotes Rally coefficient. $\vec{F}_{\text {lift, } p}$ denotes volume force, $\bar{\tau}$ denotes stress tensor, $\vec{F}_{\text {lift, } p}$ denotes lift force, $\vec{F}_{v m, p}$ stress tensor virtual mass force.

For the sparse two-phase flow in the internal flow field of impeller machinery with low-speed rotation, the lift force is unimportant relative to the drag force, so it can be ignored. But for phases which are going to separate, the lift force is important, which should be included. The particles do accelerate motion in fluid, which will drive the surround fluid accelerate, so it is virtual mass force.

\section{Discrete Method}

The discrete of equations adopts finite volume method with staggered grids. The scalars such as pressure $p$, turbulent energy $\mathrm{k}$, and turbulent energy dissipation rate $\varepsilon$ are placed on the midpoint of the grids, the velocity $\mathrm{u}$ and $\mathrm{v}$ are arranged in the center of the boundary of the grids. The discrete of convection - diffusion terms adopts a format of power function, using simple algorithm to solve the equation.

\section{Calculation Examples and Simulation Results}

The basic parameters of $\mathrm{W}$-type sewage pumps are used in numerical simulation as follows: the outlet diameter of pump is $50 \mathrm{~mm}$, the width of blade outlet is $24 \mathrm{~mm}$, flow is $15 \mathrm{~m} / \mathrm{h}$, head is $20.6 \mathrm{~m}$, speed is $2840 \mathrm{r} / \mathrm{min}$ and speed ratio is 69. Set the entire pump flow channel as the research object, for coupling calculation of volute and impeller. Set impeller centerline as y-axis, the rotation axis of the impeller as Z-axis, and the radial as X-axis. Using GAMBIT generates unstructured grids with hybrid tetrahedral mesh. Where the impeller grids are 22.0286 million, scroll grids are 9.0582 million and the generated mesh is shown in Figure 1:

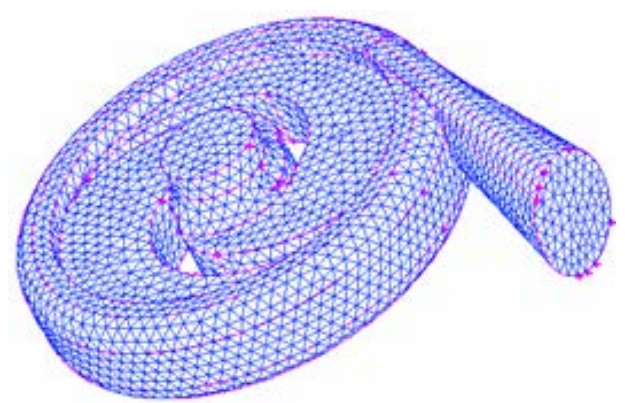

Fig 1: the generated mesh

Using standard k- $\varepsilon$ turbulence model for the solid and gas phase. Given the speed of liquid phase inlet, for the solid phase, the inlet has the same speed as the liquid phase, the diameter of solid particles and the distribution of inlet concentration. The distribution of solid particles on the inlet cross-section is uniformly, and the outlet pressure is same as liquid phase.

Liquid Boundary Conditions. On the import border, the distribution of flow speed and volume are given, assuming that the turbulent flow has been fully developed, the turbulent kinetic energy $\mathrm{K}$ and 
consumption $\varepsilon$ are: $k=\frac{3}{2}\left(u_{\text {avg }} I\right)^{2} \quad$ (5)Where , $I$ denotes Turbulence intensity $I \equiv \frac{u}{u_{\text {avg }}} \cong 0.16(\operatorname{Re})^{-1 / 8}$, $\varepsilon=C_{\mu}^{\frac{3}{4}} \frac{k^{3 / 2}}{0.07 L}$

Where $\mathrm{u}_{\mathrm{avg}}$ denotes Average speed, $\mathrm{L}$ denotes hydraulic diameter, Re denotes Reynolds, $C_{\mu}$ denotes empirical constant of the turbulence model, approximately equals to 0.09 . Import diameter for spiral, the solid wall meets the no-slip condition, i.e. the relative velocity is $\vec{W}=0$. The second boundary condition for pressure, i.e $\partial P / \partial n=0$. The wall function boundary conditions are used for the turbulent wall, while the outlet boundary conditions depending on the flow field.

The simulation results: Grit-containing water is used in calculation as the flowing medium, gravel as the solid phase, the largest volume fraction of the solid phase is $6.5 \%$ and the density is $1800 \mathrm{kG} / \mathrm{m} 3$, the results are shown as Figures 2 to 10:
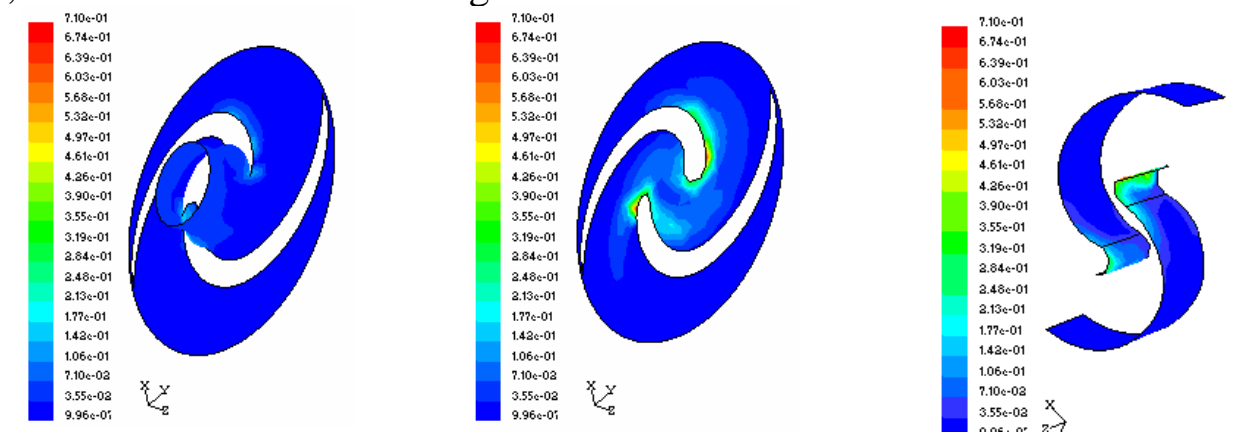

Fig 2 front cover particle distribute map Fig 3 Rear cover particle distribute map Fig 4 blade pressure surface particle distribute map $(\mathrm{Cv}=4 \%$, Particle size $4 \mathrm{~mm})$ $(\mathrm{Cv}=4 \%$, Particle size $4 \mathrm{~mm})$ $(\mathrm{Cv}=4 \%$, Particle size $4 \mathrm{~mm})$
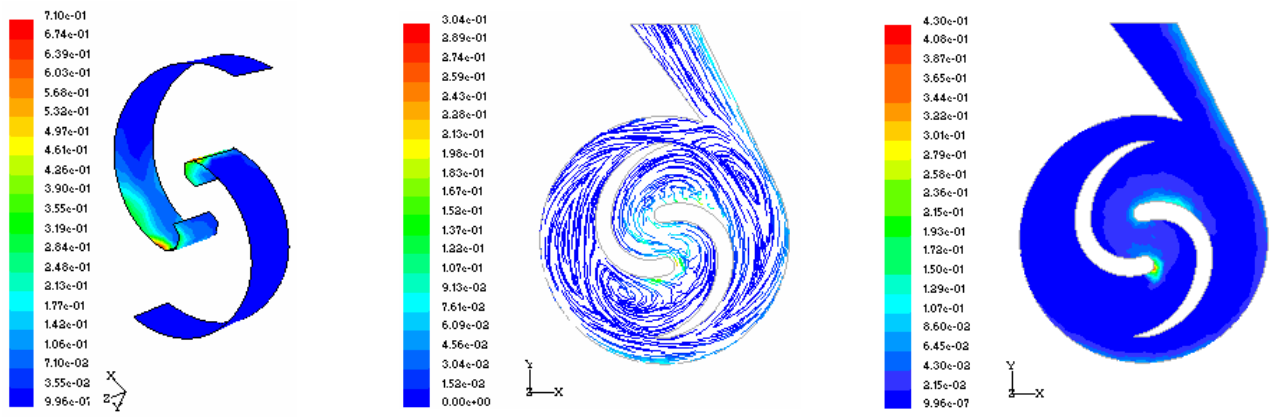

Fig 5 blade suction surface of particle distribute
\[ (\mathrm{Cv}=4 \% \text {, Particle size } 4 \mathrm{~mm}) \]
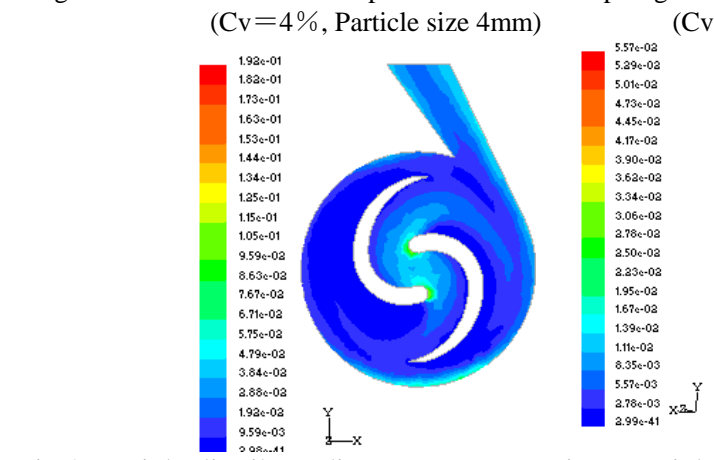
$\mathrm{Cv}=4 \%$, Particle size $5 \mathrm{~mm}$

Fig 7 Particle distribute in crossectional diagram

Fig 8 Particle distribute diagram map

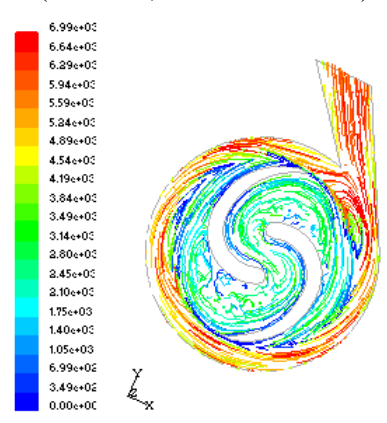

$(\mathrm{Cv}=4 \%$, Particle size $1 \mathrm{~mm})$

$(\mathrm{Cv}=1 \%$,Particle size $1 \mathrm{~mm})$

(Rinsing medium)

\section{The results and analysis}

The impact on the characters of the distribution of particles in the blade channels. The distribution characters of particles along the axial of the blade channels. The analysis of Figure 2, figure 3, Figure 7, figure 8 shown that: in the sparse two-phase flow, when conveying a higher density than solid particles of water, the particles close to the middle area of impeller back shroud and the blade channel is more than particles near the cover area of the impeller front, this trend is more obvious in the large 
particles; this suggests that the outflow of particles nears the impeller the rear cover plate. The same conclusion with the literature [3] [4].

Particles along the circumferential distribution of blade channel. The analysis of Figure 2 to figure 5, Figure 7: particles close to the middle area of impeller back shroud and the blade channel significantly closer to the pressure surface, the amount of particles near the particle far more than that of the suction surface, but the area nears the impeller front cover, this phenomenon is not so obvious; this shows that particles always along the nearby area of the blade pressure surface outflow, this conclusion is anastomotic with the literature [5].

The impact on the distribution characteristics of the particles in the blade channels of the volume concentration of particles. Distribution characteristics of the particles along the axial of the blade channels .Comparing Figure8 with Figure 9: the particles of each section increased with the increasing of particle volume concentration, but the distribution characteristics are unchanged in the axial section. Particles along the circumferential distribution of blade channel. Comparing Figure8 with Figure 9: as the increase of particle volume concentration, the particles near the exit of the pressure surface in the blade channel increase obviously.

Effect on the distribution characteristics of the particles in the blade channels. From Figure 3, figure 5, Figure 7 can be seen that: with the particle diameter increasing, the particles are close to the impeller cover plate. The bigger the particle is closer to the rear cover plate, so when transmit fluid containing large particles. The wear of the rear cover plate is more serious.

The relationship between the particle traces of pump flow passage shape. Figure 6 is the movement of solid particles by trace chart, the chart we can see that the internal flow of solid particle trajectories and the pump are identical, in sparse two-phase flow particle has a little influence on the trajectory, because the particle volume concentration is not big, the particle trajectories moving along the pressure surface under the action of gravity, the rest of the flow channel has few particle, thus the solid particle trajectory and the pump inner flow passage are identical, this conclusion is anastomotic with the literature [4].

\section{References}

[1] Jinling Lu, Guang Xi. In centrifugal pump impeller gas-liquid two-phase 3-D flow numerical study . Journal of Engineering Thermophysics, 2003 (2), 237 -240

[2] Fujun Wang. Computational fluid dynamics analysis software-CFD principle and application [M]. Beijing: Tsinghua University press, 2004

[3] Jiang Dai. study of solid-liquid two-phase turbulent flow in centrifugal pump impeller law of Beijing; Tsinghua University, 1994

[4] Jinjia Wei. Dense liquid-solid two-phase turbulent KET model and numerical calculation and experimental study of two-phase flow centrifugal pump impeller . Xi'an of Xi'an Jiao Tong University, 1998

[5] Dunmin Yang. image measurement of two-phase flow in a centrifugal pump, Journal of agricultural machinery, 2006 (12): 100 -10 